\title{
Review of: "Nanoparticles and polyaniline electrical conductivity"
}

\author{
Velram Balaji Mohan ${ }^{1}$ \\ 1 The University of Auckland
}

Potential competing interests: The author(s) declared that no potential competing interests exist.

This article compares the electrical conductivity performance of some common nanoparticles (mostly carbonic) on polyaniline (PAni). Firstly, the article is definitely interesting and informative. The interactive plots are very interesting, and VOSviewer will continue to advantage researchers in this subject matter to comprehend the research gaps and potentials. However, the article suffers from incoherence and insufficient interpretations. Also, the following further information could have been added:

1. Fabrication methods and applications of the PAni-nanofiller, which apparently decide the conductivity authors/scientists tried to achieve. Therefore, the comparison made here become somewhat inappropriate as the discussion could have highlighted this further.

2. Plus, the interaction between polyaniline and nanofiller hugely vary from their surface properties as well the functional groups. This would have been purposefully designed, like mentioned previously. 